\title{
Knowledge about HIVIAIDS among women in Bangladesh: an urban-rural comparison of trend, attitude and determinants
}

\begin{abstract}
Objective: This study is aimed to observe the knowledge trend regarding HIV/AIDS and to identify the associated factors of the HIV/AIDS knowledge among the urban and rural Bangladeshi women.
\end{abstract}

Methods: The data were extracted from the Bangladesh Demographic and Health Survey (BDHS) in 2004 and 2014, collected from women aged 15-49 years from seven administrative divisions of Bangladesh. To analyse the data, Chi-square test $\left(\boldsymbol{X}^{2}\right)$ and multivariate logistic regression analysis was utilized.

Results: In Bangladesh, the prevalence of the women's HIV/AIDS knowledge in urban area was $83.1 \%$ whereas in rural area was $63.9 \%$ in 2014 . The results revealed that reading newspaper/radio (urban: $\mathrm{aOR}=4.979, \mathrm{CI}: 2.242-11.056$ and rural: $\mathrm{aOR}=1.993$, CI: 1.372 2.889), watching TV (urban: $\mathrm{aOR}=2.129$, CI: $1.404-3.228$ and rural: $\mathrm{aOR}=2.345, \mathrm{CI}: 1.904-$ 2.889), NGO health workers visit (urban: $\mathrm{aOR}=1.592$, CI: 1.391 and rural: $\mathrm{aOR}=1.391$, CI: 1.097-1.765), women's decision-making power about health care (urban: $\mathrm{aOR}=1.486$, CI: $1.055-2.093$ and rural: $\mathrm{aOR}=1.393, \mathrm{CI}$ : 1.161-1.672) are significantly associated with having HIV knowledge as well as respondent's education, economic and marital status for both urban and rural area. Alongside with these factors, husband's education, age at marriage, condom use during intercourse, community clinic awareness, govt. health worker visits and victimization of IPV are also vital predictor of having HIV knowledge in rural Bangladesh.

Conclusion: In Bangladesh, it is essential to improve the educational program regarding HIV/AIDS especially for rural women. Mass media can play important roles in rising awareness about HIV/AIDS in both urban and rural areas. The Bangladesh Government and NGOs should increase the household visit by their health assistent and workers in this regard.

Keywords: HIV/AIDS, knowledge, determinants, logistic regression models
Volume 8 Issue 5 - 2019

\author{
Mostaured Ali Khan,' Nazrul Islam Mondal,' \\ Rafiqul Islam,' Sharif Kaikobad,' Golam \\ Mustagir,' Nafisa Tasneem² \\ 'Department of Population Science and Human Resource \\ Development, University of Rajshahi, Bangladesh \\ ${ }^{2}$ Department of Mathematics, University of Rajshahi, Bangladesh
}

Correspondence: Md. Nazrul Islam Mondal, Ph.D, Professor and Chairman, Department of Population Science and Human Resource Development, University of Rajshahi, Rajshahi-6205, Bangladesh.Email nazrulupm@gmail.com

Received: June 19, 2019 | Published: September 23, 2019
Abbreviations: HIV: human immunodeficiency virus; AIDS: acquired immune deficiency syndrome

\section{Introduction}

The infection of human immunodeficiency virus (HIV) is responsible for an incurable infectious disease named "Acquired immune deficiency syndrome (AIDS)", one of the biggest global health challenges in recent years. After identifying the first case of this chronic life-threatening ailment in the USA in 1981, the HIV/AIDS infection has permeated quickly to many countries. ${ }^{1}$ According to World Health Organization, 36.9 million people were living with HIV in 2017 , especially, women are in greater risk constituting $51 \%$ of all adults living with HIV, increasing at a rate of 7000 young women become infected per week. ${ }^{2,3}$ Besides, South-East Asian countries contain 2nd highest amount of HIV infections, 3.5 million people, right after the Africa. ${ }^{3}$ The first case of HIV/AIDS was reported in
Bangladesh in 1989. In 2011, the number of HIV-positive people was 2,533 but the amount has dramatically increased in last six years and stand at around 13000 infected people with around 1700 new cases in $2017 .{ }^{3,4}$ and $34 \%$ of which are females aged 15 and over. ${ }^{2}$ HIV/AIDS can transmit person to person through sexual contact with an infected person and through infected blood transmition. ${ }^{5}$

Though Bangladesh is not in such an alarming state of HIV epidemic, the country has greater risk beacuse of several factors: the geographical imminense with India and Myanmer, top two severely HIV infected countries in this region, ${ }^{6,7}$ gender disparity and poverty, drug trafficing and abuse, poor health-seeking behaviour and specifically, poor knowledge and awarenss about HIV/AIDS. ${ }^{7}$ ${ }^{8}$ In 2018, nearly a million of Rohingya have eluded to Bangladesh after experiencing genocide in their native land Myanmar and a good amount of them were HIV positive which increases the risk at high level. ${ }^{9}$ The infection proportions inside the susceptible clusters are 
growing, foremost, to an almost half likelihood that the worm will feast on the overall people. ${ }^{10-12}$ The circumstances have elevated thoughtful anxieties among management, government and policy makers and they are looking forward to the community knowledge and awareness on the transmission and deterrence of HIV. ${ }^{13}$

Worldwide, a wide range of researches have been conducted regarding the epidemiology, etiology and community knowledge and awareness of HIV/AIDS. Females from the growing age ( $<25$ years) are the most vulnerable to HIV knowledge. ${ }^{8,14}$ Couple's education is also vital influencing factor to have good HIV knowledge. ${ }^{15,16}$ Besides, prior studies have demonstrated that level of HIV knowledge tends to be higher in societies where people enjoy greater degree in freedom of communication, seek health advocacy, and engage in peer communication. ${ }^{16}$

Bangladeshi women's HIV/AIDS knowledge level are very poor and have a higher risk of HIV infection. ${ }^{8}$ Sometimes, a teenager was misused by the sexual ferocity and abuse, initial sexual beginning and they do not have the ability to negotiate for safe sex, and also other factors such as the absence of education. ${ }^{17}$ Again, women living in rural areas are less likely to have extensive knowledge about HIV compared to urban women. ${ }^{8,18,19}$ Existing literature regarding urban and rural inequalities as they appertain to HIV/AIDS knowledge in Bangladesh is very limited. Therefore, comparing the rural-urban trend, this study is aimed to notice the attitudes towards HIV/ AIDS among women and to identify the associated factors with the knowledge and awareness about HIV/AIDS among the urban and rural Bangladeshi women.

\section{Data and Methodology}

\section{Study setting and sampling procedure}

To conduct this study, two waves of national representative crosssectional data were extracted from Bangladesh Demographic and Health Survey (BDHS) conducted in 2004 and 2014. These two surveys were conducted in the seven administrative divisions of Bangladesh: Dhaka, Chittagong, Rajshahi, Khulna, Sylhet, Rangpur, and Barisal. The District (zilas) is the part of one division and are divided into further administrative unit called "Upazilas", consisting of urban (wards and city corporation unit) and rural areas (Union parishad and villages). A sampling frame for the list of enumeration areas (EAs) in these surveys were utilized based on the national population and housing census in 2001 and 2011 by Bangladesh Bureau of Statistics (BBS) ${ }^{20,21}$ In these surveys, the household was selected based on a two-stage stratified technique. In the $1^{\text {st }}$ stage, a total of 361 for BDHS-2004 and 600 for BDHS-2014 of enumeration areas consisting of urban (122 in BDHS-2004 and 207 in BDHS2014) and rural areas (239 in BDHS-2004 and 393 in BDHS-2014) were selected with the probability proportional size. In the 2nd stage, households were independently selected from the urban and rural areas from the 7 divisions using the systematic sampling, based on demographic and health variables. The study design has selected 10,811 and 18,000 residential households for the survey in 2004 and 2014, respectively. ${ }^{20,21}$ The overall response rate was $99 \%$. Total sample size of these surveys were 11440 in 2004 and 17863 in 2014. All information was collected from women at reproductive age (1549 years). In this study analysis, we ulitized total sample size of these surveys in particular year due to there was no missing values in outcome variable.

\section{Response and explanatory variables}

To fulfil the study objective, respondent's knowledge about HIV/ AIDS was selected as response or dependent variable gathered by asking question, "Have Knowledge about HIV/AIDS", is addressed as follows, $Y=$ Have Knowledge about $H I V=\left\{\begin{array}{l}1, Y e s \\ 0, N o\end{array} ;\right.$ Further the respondents were asked few more questions to observe their level of knowledge and notion about HIV.

On the other hand, various socio-demographic variables were used as explanatory variables to identify the influencing factors of the knowledge about HIV/AIDS in urban-rural differential context. These variables were selected in accordance with their importance based on previous studies. Explanatory variable includes: respondents' age, education, husband's education, economic status, geographical variance, marital status, age at marriage, number of children, current contraceptive use, reading newspaper/radio, watching television, visited community clinic, visited by government health workers, visited by NGO workers, victim of intimate partner violence and decision-making power about health care.

\section{Statistics analysis}

The data was analysed using Statistical Program for Social Sciences (SPSS) in windows verison25. To calculate relative

change in HIV/AIDS knowledge from 2004 to 2014, the following formula was used: $\frac{P_{2014}-P_{2004}}{P_{2004}} \times 100$. To assess the association with knowledge about HIV/AIDS and some explanatory variables, the chi-square $\left(\chi^{2}\right)$ test was conducted (set up for significant at $\rho<0.05$ ). The associations between the knowledge about HIV/AIDS and the possible influencing factors were examined with logistic regression models. As the outcome variable was dichotomized in binary form, the binary logistic regression analysis was used to identify the risk factors. The odds ratios were estimated and to measure the level of significance, 95\% Confidence Interval (CI) was also estimated at $\rho<0.05$ of significance level.

\section{Results}

In Bangladesh, the prevalence of the women's HIV/AIDS knowledge in urban area was $83.1 \%$ in 2014 with a rate of $1.71 \%$ increase whereas in rural area had $63.9 \%$ in 2014 with a rate of $18.9 \%$ increase over the period 2004-2014. Table 1 represents the relative change in HIV/AIDS knowledge in both urban and rural area according to various socio-demographic and economic characteristics over the period 2004 to 2014. The highest increase of HIV knowledge observed in age group 35-49 years (urban: $14.8 \%$ and rural: $50.9 \%$ ), and among urban and rural poorest women, $13.8 \%$ and $54.9 \%$, respectively. Muslim and employed rural women had $21.7 \%$ and $29.5 \%$ increase in HIV knowledge over last 10 years, respectively. The highest increase of HIV knowledge was found in rural area of Sylhet division (39.8\%). 
Table I: Relative change in knowledge about HIVIAIDS according to socio-demographic and economic characteristics over the period 2004-20I4 in Bangladesh

\begin{tabular}{|c|c|c|c|c|c|c|}
\hline \multirow{2}{*}{ Background Characteristics } & \multicolumn{3}{|l|}{ Urban } & \multicolumn{3}{|l|}{ Rural } \\
\hline & $2004(\%)$ & $2014(\%)$ & Change (\%) & $2004(\%)$ & $2014(\%)$ & Change (\%) \\
\hline Overall HIV Knowledge & 81.7 & 83.1 & I.7I & 53.7 & 63.9 & 18.9 \\
\hline \multicolumn{7}{|l|}{ Age (in years) } \\
\hline $15-24$ & 86.1 & 83.9 & -2.6 & 65.6 & 64.0 & -2.4 \\
\hline $25-34$ & 84.1 & 82.6 & -1.8 & 52.0 & 63.7 & 22.5 \\
\hline $35-49$ & 74.9 & 86.0 & 14.8 & 42.4 & 64.0 & 50.9 \\
\hline \multicolumn{7}{|l|}{ Education } \\
\hline No education & 62.3 & 59.1 & -5.3 & 30.5 & 33.8 & 10.8 \\
\hline Primary' & 80.3 & 75.8 & -5.6 & 57.8 & 58.3 & 0.9 \\
\hline Secondary and above' & 97.2 & 94.5 & -4.2 & 85.7 & 86.4 & 0.5 \\
\hline \multicolumn{7}{|l|}{ Economic status $^{2}$} \\
\hline Poorest & 43.6 & 49.6 & 13.8 & 27.3 & 42.3 & 54.9 \\
\hline Poor & 60.3 & 66.4 & 10.1 & 41.9 & 55.2 & 31.7 \\
\hline Middle class & 69.9 & 75.4 & 7.9 & 56.2 & 70.7 & 25.8 \\
\hline Rich & 82.0 & 83.9 & 2.3 & 73.0 & 80.2 & 9.9 \\
\hline Richest & 95.0 & 93.9 & -1.1 & 86.7 & 91.3 & 5.3 \\
\hline \multicolumn{7}{|l|}{ Religion } \\
\hline Muslim & 81.5 & 83.3 & 2.2 & 53.0 & 64.5 & 21.7 \\
\hline Non-Muslim & 82.7 & 81.0 & -2.1 & 59.4 & 57.5 & -3.2 \\
\hline \multicolumn{7}{|l|}{ Employment status } \\
\hline Unemployed & 84.0 & 84.3 & 0.4 & 54.8 & 63.8 & 16.4 \\
\hline Employed & 74.6 & 83.8 & 12.3 & 49.5 & 64.1 & 29.5 \\
\hline \multicolumn{7}{|l|}{ Division } \\
\hline Barisal & 80.6 & 87.2 & 8.2 & 59.2 & 66.8 & 12.8 \\
\hline Chittagong & 76.7 & 80.5 & 4.9 & 54.0 & 66.1 & 22.4 \\
\hline Dhaka & 87.1 & 88.5 & 1.6 & 56.1 & 69.3 & 23.5 \\
\hline Khulna & 90.3 & 87.8 & -2.8 & 67.3 & 74.3 & 10.4 \\
\hline Rajshahi & 72.8 & 82.1 & 12.8 & 46.3 & 58.5 & 26.4 \\
\hline Sylhet & 80.5 & 74.5 & -7.5 & 40.0 & 55.9 & 39.8 \\
\hline
\end{tabular}

Note: $\quad$ 'Primary and secondary education is defined as completing grade 5 and 10 , respectively

${ }^{2}$ Followed BDHS wealth index standard measure

\section{People's attitude towards HIV}

Urban-rural knowledge differentials of HIV/AIDS are represented in Table 2 . The study found, $63.6 \%$ and $75.4 \%$ of urban women, and $56.6 \%$ and $70.3 \%$ of rural women answered condom use during sexual intercourse and intercourse with only one partner rather than multiple can reduce the risk of AIDS, respectively. Surprisingly, 37.1\% of rural women even $27.1 \%$ of urban women still believed that HIV can spread from mosquito bites and many other prejudices like HIV can spread by sharing food with an infected person (rural: $42.8 \%$ and urban: $31.2 \%$ ) or it's a witchcraft. On the other hand, women had good knowledge that unsafe blood transmission and unsterilized syringe use $(90.3 \%$ and $90.7 \%$ of urban women and $86.5 \%$ and $89.0 \%$ of rural women, respectively) increases the infection risk highly. Besides, $62.5 \%$ of rural women and $62.7 \%$ of urban women assumed that HIV can transmit during delivery and $78.3 \%$ of rural women and $79.7 \%$ of urban women deemed that HIV can transmit by breastfeeding. 
Table 2: Urban-rural knowledge differentials about HIVIAIDS in Bangladesh in 20I4

\begin{tabular}{lll}
\hline \multirow{2}{*}{ Knowledge items } & Year 20 I4 & \\
\cline { 2 - 2 } & Urban (\%) & Rural (\%) \\
\hline To reduce the risk of HIV & \multicolumn{2}{l}{5} \\
Use condom during sex & 63.6 & 56.6 \\
Have one sex partner only & 75.4 & 70.3 \\
Prejudice & & \\
Spread through mosquito bites & 27.1 & 37.1 \\
Spread by sharing food with infected person & 31.2 & 42.7 \\
Can be infected by witchcraft & 8.6 & 11.6 \\
HIV can get through & & \\
Using unsterilized needle or syringe & 90.7 & 89.0 \\
Unsafe blood transmission & 90.3 & 86.5 \\
HIV can transmit & & \\
During pregnancy & 80.7 & 79.7 \\
During delivery & 62.7 & 62.5 \\
By breastfeeding & 79.7 & 78.3 \\
\hline
\end{tabular}

\section{Associated risk factors}

Table 3 represents the association between HIV/AIDS knowledge and various explanatory variables in the urban and rural Bangladesh (BDHS 2014) were assessed by the $\chi^{2}$-test. Respondent's age (35-49 years: $86 \%$ ) was significantly associated with HIV/AIDS knowledge in the urban area but not in the rural area. Showing a highly significant association, secondary or above educated Bangladeshi women had highest HIV knowledge in both urban and rural area $(94.5 \%$ and $76.2 \%$ respectively) but illiterate rural women had a very poor knowledge (only 33.8\%). The study result showed that the HIV knowledge was significantly higher among rich (urban: $83.9 \%$ and rural: $80.2 \%$ ) and richest (urban: $93.9 \%$ and rural: 91.3\%) family. The HIV/AIDS knowledge was significantly higher among married women, whose age at marriage was more than 16 year in both areas and use condom as contraceptive method. Media exposer like reading newspaper/ radio, watching television was also a good associated factor. Among the Bangladeshi rural women, community clinic visit $(66.9 \%)$ and visited by goverment health workers $(75.2 \%)$ are playing positive role on having HIV knowledge. The knowldege was significant higher among women who were visited by NGO worlers and have decision making power in the family, and lower among women who were vitim of initimate partner violence in both area.

Table 3: Associated factors of having knowledge about HIV/AIDS within Bangladesh in 2014

\begin{tabular}{|c|c|c|c|c|c|c|}
\hline \multirow{3}{*}{$\begin{array}{l}\text { Explanatory } \\
\text { Variables }\end{array}$} & \multicolumn{6}{|c|}{ Knowledge about HIVIAIDS } \\
\hline & \multicolumn{2}{|l|}{ Urban } & \multirow{2}{*}{$p$-value } & \multicolumn{2}{|l|}{ Rural } & \multirow{2}{*}{$p$-value } \\
\hline & No & Yes & & No & Yes & \\
\hline \multicolumn{7}{|l|}{ Age (in years) } \\
\hline $15-24$ & $264(16.1 \%)$ & |374(83.9\%) & & $1236(36.0 \%)$ & $2198(64.0 \%)$ & \\
\hline $25-34$ & $342(17.4 \%)$ & $1623(82.6 \%)$ & 0.016 & $1502(36.3 \%)$ & $2639(63.7 \%)$ & 0.962 \\
\hline $35-49$ & $267(14.0 \%)$ & $1635(86.0 \%)$ & & $1350(36.0 \%)$ & $2397(64.0 \%)$ & \\
\hline \multicolumn{7}{|l|}{ Education } \\
\hline No education & $472(40.9 \%)$ & $681(59.1 \%)$ & & $202 I(66.2 \%)$ & 1032(33.8\%) & \\
\hline Primary' & $379(24.2 \%)$ & $1187(75.8 \%)$ & $<0.001$ & $1527(4 \mid .7 \%)$ & $2133(58.3 \%)$ & $<0.001$ \\
\hline Secondary and above' & $191(5.5 \%)$ & $3257(94.5 \%)$ & & $680(\mid 3.6 \%)$ & $4303(86.4 \%)$ & \\
\hline \multicolumn{7}{|l|}{ Husband's education } \\
\hline No education & $48 I(37.4 \%)$ & $805(62.6 \%)$ & & $2136(56.6 \%)$ & $1640(43.4 \%)$ & \\
\hline Primary & $336(23.8 \%)$ & $1075(76.2 \%)$ & $<0.001$ & $1326(38.5 \%)$ & $2118(61.5 \%)$ & $<0.001$ \\
\hline Secondary \& above & $225(6.5 \%)$ & $3244(93.5 \%)$ & & $766(17.1 \%)$ & $3708(82.9 \%)$ & \\
\hline
\end{tabular}


Table Continued...

\begin{tabular}{|c|c|c|c|c|c|c|}
\hline \multicolumn{7}{|c|}{ Economic status } \\
\hline Poorest & $263(50.4 \%)$ & $259(49.6 \%)$ & $<0.001$ & I575(57.7\%) & II54(42.3\%) & \multirow{5}{*}{$<0.001$} \\
\hline Poor & $139(33.6 \%)$ & $275(66.4 \%)$ & & $|32|(44.8 \%)$ & $1625(55.2 \%)$ & \\
\hline Middle & $206(24.6 \%)$ & $630(75.4 \%)$ & & $815(29.3 \%)$ & 1970(70.7\%) & \\
\hline Rich & $266(16.1 \%)$ & $1385(83.9 \%)$ & & $420(19.8 \%)$ & 1698(80.2\%) & \\
\hline Richest & $168(6.1 \%)$ & $2576(93.9 \%)$ & & $97(8.7 \%)$ & $1021(91.3 \%)$ & \\
\hline \multicolumn{7}{|l|}{ Marital status } \\
\hline Married & $916(16.0 \%)$ & $4823(84.0 \%)$ & \multirow[t]{2}{*}{$<0.001$} & $3914(35.3 \%)$ & $7177(64.7 \%)$ & \multirow[t]{2}{*}{$<0.001$} \\
\hline Others & $126(29.4 \%)$ & $302(70.6 \%)$ & & $314(51.9 \%)$ & $291(48.1 \%)$ & \\
\hline \multicolumn{7}{|c|}{ Age at marriage (in years) } \\
\hline$\leq 16$ & $773(21.4 \%)$ & $2833(78.6 \%)$ & \multirow[t]{2}{*}{$<0.001$} & $3276(40.5 \%)$ & $4807(59.5 \%)$ & \multirow[t]{2}{*}{$<0.001$} \\
\hline$>16$ & $269(10.5 \%)$ & $2292(89.5 \%)$ & & $952(26.3 \%)$ & $266 \mid(73.7 \%)$ & \\
\hline \multicolumn{7}{|c|}{ Number of children } \\
\hline$\leq 2$ children & $5 \mid 8(\mid 5.6 \%)$ & $2792(84.4 \%)$ & \multirow[t]{2}{*}{0.603} & $2365(35.8 \%)$ & $4240(64.2 \%)$ & \multirow[t]{2}{*}{0.431} \\
\hline$>2$ children & $355(16.2 \%)$ & $1840(83.8 \%)$ & & $1723(36.5 \%)$ & $2994(63.5 \%)$ & \\
\hline \multicolumn{7}{|c|}{ Current contraceptive use } \\
\hline Not using & $493(20.5 \%)$ & $1912(79.5 \%)$ & & $1943(39.0 \%)$ & $3043(61.0 \%)$ & \multirow{3}{*}{$<0.001$} \\
\hline Condom & $28(4.2 \%)$ & $635(95.8 \%)$ & \multirow[t]{2}{*}{$<0.001$} & $55(12.3 \%)$ & $391(87.7 \%)$ & \\
\hline Other methods & $521(16.8 \%)$ & $2578(83.2 \%)$ & & $2230(35.6 \%)$ & $4034(64.4 \%)$ & \\
\hline \multicolumn{7}{|c|}{ Reading newspaper/radio } \\
\hline Not at all & $987(21.7 \%)$ & $356 \mid(78.3 \%)$ & \multirow{2}{*}{$<0.001$} & $4078(40.2 \%)$ & $6064(59.8 \%)$ & \multirow{2}{*}{$<0.001$} \\
\hline Yes & $53(3.3 \%)$ & $1560(96.7 \%)$ & & $142(9.3 \%)$ & $1389(90.7 \%)$ & \\
\hline \multicolumn{7}{|c|}{ Watching television } \\
\hline Not at all & $477(43.2 \%)$ & $626(56.8 \%)$ & \multirow{2}{*}{$<0.001$} & $3097(51.9 \%)$ & $2867(48.1 \%)$ & \multirow{2}{*}{$<0.001$} \\
\hline Yes & $565(\mid 1.2 \%)$ & $4499(88.8 \%)$ & & $1130(19.7 \%)$ & $460 I(80.3 \%)$ & \\
\hline \multicolumn{7}{|c|}{ Visited community clinic } \\
\hline No & $896(17.1 \%)$ & $4358(82.9 \%)$ & \multirow{2}{*}{0.429} & $2810(39.9 \%)$ & $4598(60.1 \%)$ & \multirow{2}{*}{$<0.001$} \\
\hline Yes & $146(16.0 \%)$ & 767(84.0\%) & & $1418(33.1 \%)$ & $2870(66.9 \%)$ & \\
\hline \multicolumn{7}{|c|}{ Visited by government health workers } \\
\hline No & $206(16.0 \%)$ & $1083(84.0 \%)$ & \multirow[t]{2}{*}{0.956} & $855(34.2 \%)$ & 1644(65.8\%) & \multirow[t]{2}{*}{$<0.001$} \\
\hline Yes & $26(16.1 \%)$ & $135(83.9 \%)$ & & I 35 (24.8\%) & $409(75.2 \%)$ & \\
\hline Visited by NG & & & & & & \\
\hline Not at all & $187(17.4 \%)$ & $887(82.6 \%)$ & 0.013 & $835(34.2 \%)$ & $1610(65.8 \%)$ & $<0.001$ \\
\hline Yes & $45(12.0 \%)$ & $331(88.0 \%)$ & & $155(25.9 \%)$ & $443(74.1 \%)$ & \\
\hline Victim of intin & tner violenc & & & & & \\
\hline No & $667(\mid 4.5 \%)$ & $393 \mid(85.5 \%)$ & $<0.001$ & $2533(32.0 \%)$ & $5384(68.0 \%)$ & $<0.001$ \\
\hline Yes & $36 I(23.9 \%)$ & $1149(76.1 \%)$ & & $1607(44.4 \%)$ & $20 \mid 3(55.6 \%)$ & \\
\hline Decision maki & er about hea & & & & & \\
\hline Do not have & $404(21.2 \%)$ & $1505(78.8 \%)$ & $<0.001$ & $1674(39.1 \%)$ & $2603(60.9 \%)$ & $<0001$ \\
\hline Have & $5 \mid 2(13.4 \%)$ & $3318(86.6 \%)$ & & $2240(32.9 \%)$ & $4574(67.1 \%)$ & -0.001 \\
\hline
\end{tabular}

Note: NGO, Non-Government Organization

\section{Identification of determinants}

Table 4 shows that women education is a vital factor of having knowledge about HIV/AIDS in both urban and rural Bangladesh. Females who have finished at least secondary education had 4.048 (CI: 2.438-6.721) times more knowledge about HIV/AIDS than the illiterate urban women. On the other hand, in rural areas, the likelihood of having HIV/AIDS knowledge was increased among women who had completed primary $(\mathrm{aOR}=2.105$, CI: $1.619-2.737)$ and secondary or higher education $(\mathrm{aOR}=4.190, \mathrm{CI}$ : $3.160-5.556)$ than the illiterate women, respectively and also increased for rural women with husband who had completed secondary or higher 
education $(\mathrm{aOR}=1.487, \mathrm{CI}: 1.143-1.934)$ than the women with illiterate husband. The women from middle class (urban: $\mathrm{aOR}=1.482$, CI: 0.828-2.656; rural: aOR=1.671, CI: 1.277-2.187), rich (urban: $\mathrm{aOR}=2.623$, CI: $1.492-4.611$; rural: $\mathrm{aOR}=2.136$, CI: $1.526-2.991)$ and richest families (urban: $\mathrm{aOR}=4.365$, CI: 2.270-8.394; rural: $\mathrm{aOR}=3.6$, CI: 1.989-6.517) have greater likelihood of having knowledge of HIV/ AIDS than the poor women in both areas. The likelihood of having HIV knowledge was decreased among women of other marital status rather than married (urban: $\mathrm{aOR}=0.455, \mathrm{CI}: 0.365-0.567$ and rural: $\mathrm{aOR}=0.505$, CI: 0.429-0.596). In rural area, the chance of having HIV knowledge was increased among women of $>16$ years age at marriage $(\mathrm{aOR}=1.331$, CI: 1.096-1.615) than the women who got married at $\leq 16$ years of age and among couples who use condom $(\mathrm{aOR}=2.151$, CI: 1.154-4.011) as contraceptive method than contraceptive nonusers. The likelihood of having knowledge was increased among newspaper/radio readers (urban: $\mathrm{aOR}=4.979, \mathrm{CI}: 2.242-11.056$ and

Table 4: Logistic regression analysis to estimate the determinants of having knowledge about HIV/AIDS in Bangladesh in 2014

\begin{tabular}{|c|c|c|c|c|c|c|}
\hline \multirow{3}{*}{ Explanatory variables } & \multicolumn{3}{|l|}{ Urban } & \multicolumn{3}{|l|}{ Rural } \\
\hline & \multirow{2}{*}{$\begin{array}{l}\text { Adjusted } \\
\text { OR }\end{array}$} & \multicolumn{2}{|l|}{$95 \% \mathrm{Cl}$} & \multirow{2}{*}{$\begin{array}{l}\text { Adjusted } \\
\text { OR }\end{array}$} & \multicolumn{2}{|l|}{$95 \% \mathrm{Cl}$} \\
\hline & & Lower & Upper & & Lower & Upper \\
\hline \multicolumn{7}{|l|}{ Education } \\
\hline No education ${ }^{(\mathrm{R})}$ & 1.00 & $\ldots \ldots$. & $\ldots \ldots$. & 1.00 & $\ldots \ldots$. & $\ldots \ldots$. \\
\hline Primary & 1.435 & 0.908 & 2.344 & $2.105^{\text {*tok }}$ & 1.619 & 2.737 \\
\hline Secondary \& Above & $4.048^{\text {*ink }}$ & 2.438 & 6.721 & $4.190^{\text {sopk }}$ & 3.160 & 5.556 \\
\hline \multicolumn{7}{|l|}{ Husband's education } \\
\hline No education ${ }^{(R)}$ & 1.00 & $\ldots \ldots$ & $\ldots \ldots$ & 1.00 & $\ldots \ldots$ & $\ldots \ldots$ \\
\hline Primary & 1.072 & 0.691 & 1.663 & 1.062 & 0.850 & 1.328 \\
\hline Secondary \& Above & 1.355 & 0.835 & 2.198 & $1.487^{* 0 k}$ & 1.143 & 1.934 \\
\hline \multicolumn{7}{|l|}{ Economic status } \\
\hline Poorest ${ }^{(\mathrm{R})}$ & 1.00 & $\ldots \ldots$. & $\ldots \ldots$ & 1.00 & $\ldots \ldots$ & $\ldots \ldots$ \\
\hline Poor & 1.402 & 0.767 & 2.564 & 1.199 & 0.954 & 1.507 \\
\hline Middle & $1.482^{*}$ & 0.828 & 2.656 & $\left.1.67\right|^{* 00 k}$ & 1.277 & 2.187 \\
\hline Rich & $2.623^{* * * *}$ & 1.492 & 4.611 & $2.136^{\text {woth }}$ & 1.526 & 2.991 \\
\hline Richest & 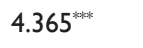 & 2.270 & 8.394 & $3.600^{* 00 k}$ & 1.989 & 6.517 \\
\hline \multicolumn{7}{|l|}{ Marital status } \\
\hline Married (R) & 1.00 & $\ldots \ldots$ & $\ldots \ldots$ & 1.00 & $\ldots \ldots$ & $\ldots \ldots$ \\
\hline Others & $0.455^{\text {*⿻*⿲丿丨丨二 }}$ & 0.365 & 0.567 & $0.505^{\text {*od }}$ & 0.429 & 0.596 \\
\hline \multicolumn{7}{|c|}{ Age at marriage (in years) } \\
\hline$\leq 16^{(R)}$ & 1.00 & $\ldots \ldots$ & $\ldots \ldots$ & 1.00 & $\ldots \ldots$ & $\ldots \ldots$ \\
\hline$>16$ & 1.358 & 0.942 & 1.959 & $1.331^{* *}$ & 1.096 & 1.615 \\
\hline \multicolumn{7}{|c|}{ Current contraceptive use } \\
\hline Not using ${ }^{(R)}$ & 1.00 & $\ldots \ldots$. & $\ldots \ldots$. & 1.00 & $\ldots \ldots$. & $\ldots \ldots$. \\
\hline Condom & 1.327 & 0.639 & 2.880 & $2.151^{* *}$ & 1.154 & 4.011 \\
\hline Other methods & 1.157 & 0.792 & 1.692 & 1.096 & 0.907 & 1.325 \\
\hline \multicolumn{7}{|c|}{ Reading newspaper/radio } \\
\hline Not at all ${ }^{(R)}$ & 1.00 & $\ldots \ldots$. & $\ldots \ldots$. & 1.00 & $\ldots \ldots$. & $\ldots \ldots$. \\
\hline Yes & $4.979^{* * *+1}$ & 2.242 & 11.056 & $1.993^{\text {*opk }}$ & 1.372 & 2.889 \\
\hline
\end{tabular}

Citation: Khan MA, Mondal NI, Islam R, et al. Knowledge about HIV/AIDS among women in Bangladesh: an urban-rural comparison of trend, attitude and determinants. Biom Biostat Int J. 2019;8(5):I62-170. DOI: 10.15406/bbij.2019.08.00285 rural: $\mathrm{aOR}=1.993, \mathrm{CI}: 1.372-2.889)$ and who watch television (urban: $\mathrm{aOR}=2.129$, CI: $1.404-3.228$ and rural: $\mathrm{aOR}=2.345$, CI: $1.904-2.889$ ) frequently. Besides, rural women who visited community clinic have 1.2 times (aOR=1.2, CI: 0.995-1.448), were visited by government health workers have 1.509 times $(\mathrm{aOR}=1.509$, CI: $1.178-1.934)$ higher knowledge regarding HIV. Similarly, the likelihood of having knowledge about HIV/AIDS was increased among women who were visited by $\mathrm{NGO}$ workers (urban: $\mathrm{aOR}=1.592$, CI: 1.391 and rural: aOR=1.391, CI: 1.097-1.765) and who had decision making power about health care (urban: $\mathrm{aOR}=1.486$, CI: $1.055-2.093$ and rural: $\mathrm{aOR}=1.393$, CI: $1.161-1.672$ ) in the family than the women without NGO worker visit and decision making power in the family, respectively. In the rural area, victims of IPV have $0.795(\mathrm{aOR}=0.795$, CI: 0.656-0.963) times lower chances to have HIV knowledge than the women who were not IPV victim. 
Table Continued...

\section{Watching television}

Not at all ${ }^{(R)}$

$$
1.00
$$

Yes

$2.129^{\text {mate }}$

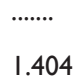

3.228

1.00

Visited community clinic

No ${ }^{(R)}$

1.00

Yes

0.866

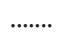

$\ldots \ldots .$.

1.361

........

2.430

1.00

0.849

........

1.061

2.388

$$
1.00
$$

$1.391^{* *}$

1.097

1.765

Victim of intimate partner violence (IPV)

No ${ }^{(R)}$

Yes

1.00

0.801

…...

…...

1.00

0.555

1.154

$0.795^{\text {** }}$

0.656

0.963

Decision making power about health care

Do not have ${ }^{(\mathrm{R})}$

1.00

.......

.......

1.00

Have

$1.486^{*}$

1.055

2.093

$1.393^{\text {**to }}$

1.161

1.672

Note: “(R)” denotes reference category and significance at $* * * p<0.001, * * p<0.01$ and $*_{p}<0.05$ levels

$\mathrm{OR}$, odds ratio; $\mathrm{Cl}$, confidence interval

\section{Discussion}

In Bangladesh, a little increase of HIV/AIDS knowledge is observed in urban area whereas in rural area, it shows a good rising trend though the proportion of women with HIV/AIDS knowledge is much lower in this cluster than urban areas. Different socio-economic and demographic variables tragedies play a vital role in prompting HIV/AIDS. However, it is difficult in rural Bangladesh to rise HIV awareness through accurate knowledge, because Bangladesh has needed to develop the educational condition in poor rural areas and have poor believe and knowledge about HIV than urban women, even though there still exist prejudices. According to this study result, both urban and rural women have good level of knowledge about HIV transmission. Almost every woman knows that use of unsterilized syringe, unsafe blood transmission highly increases the risk of HIV infection. They also have good knowledge regarding mother to child HIV transmission process. But it is saddening but truth is, a good number of rural women do not agree with condom use can reduce the risk of HIV infection and they still believes in prejudice like HIV is a witchcraft, can transmit through mosquito bites etc. Additionally, a very few prejudices also exist in urban areas. Prior endeavors have a significanlty higher HIV prevalence is observed among people who are unaware and have poor knowledge of the potential routes of HIV transmission. ${ }^{16,22}$ If adequate knowledge regarding HIV transmission and prevention is ensured then it will be very easy to reduce the prevalecnce of HIV/AIDS in our country.

However, the study results indicate that in both urban and rural area, women aged 35-49 years have a comparatively better HIV knowledge and especially the trend is rising among rural women. Females from the growing age ( $<25$ years) are unprotected from fresh skills, connecting to sexuality and imitation. ${ }^{14}$ which increases the risk of HIV infection. The deprived knowledge and awareness in the fresher cluster ( $<25$ years) may be owing to the old-style community scheme especially in rural community that often disregard this cluster as a distinct unit who is preserved, neither as a kid nor as mature. In this regard, the women's education has an immense impact on having better HIV/AIDS knowledge especially in rural area. Our study results regarding higher odds for educated women in showing consistency with several earlier studies. ${ }^{16,23}$ A prior study has termed education as "social vaccine" against HIV/AIDS. ${ }^{16}$ In both urban and rural area, a very good HIV knowledge in educated cluster is observed over the year 2004 to 2014 but illiterate rural women are still now struggling for poor knowledge. In this regard, Mondal et al. ${ }^{15}$ demonstrated that women with educated husband have higher chance to have better HIV knowledge.

Economic condition of women is a very important predictor of having HIV knowledge. In rural Bangladesh, a very high increasing knowledge trend of HIV is observed in poor community. This study results regarding higher likelihood is supporting prior studies. Besides, Mondal et al. ${ }^{24}$ uncovered a positive association between economic status and misconception about HIV, poor household tends to more misconception in Bangladesh.

In rural Bangladesh, there is an intension is still prevailing to get female adolescents married at age $<16$ years. A lower odds of having HIV knowledge is revealed among women belongs to this category is supported by previous literature. ${ }^{25}$ Furthermore, a prior endeavour has identified a significant impact of age at marriage on HIV epidemic in Japan. ${ }^{26}$ Contraceptive use during sexual intercourse and proprer family planning knowledge have been proven to be lucrative for not 
only to protect unwanted birth but also from sexually transmitted diseases like HIV/AIDS. ${ }^{27,}{ }^{28}$ This study reports that rural women who use condom as contraceptive during intersourse are more likely to have HIV knowledge compared to the non-contraceptive user. This finding is in agreement with the other studies. ${ }^{15,27}$ In rural areas, there is an unwillingness of contraceptive use always remain due to religious orthodoxy.

Mass communication disclosure is a very important factor associated with the HIV knowledge. Females with entrance to mass communication (television, radio, newspapers etc.) have the chance to divide into their opinions and philosophies through their families and so this type of women have more knowledge and awareness about HIV/AIDS. According to this study report, women who got media exposure have a higher likelihood of knowledge about HIV/ AIDS in both urban and rural area which is supported by preceding literatures. ${ }^{7,8,29}$ The publicity to such media can interconnect knowledge and consciousness about HIV/AIDS through the tune, newscast information, theatres, and movies that can intensely affect the approach and behaviour of the public. ${ }^{14}$ Besides, media can positively influence condom use practice during intercourse which can reduce HIV/AIDS risk. ${ }^{29}$ The accessibility of media exposure is always higher in urban sector than the rural. So, social awareness program is specially needed in rural areas to implement in this regard as well as urban sector.

However, community clinic awareness, government and NGO health worker visits are also playing good role on increasing HIV knowledge in rural Bangladesh. This study identifies a higher likelihood of having knowledge is associated with women who were aware of community clinic, were visited by government and NGO health workers. Rural women can get informed about the etiology and transmission mode of HIV from these sources which helps AIDS prevention. Also, regular health worker visit can make women aware and prevent HIV infection. This study also found a positive association between IPV victimization and women's decision-making power in family about health care, and HIV knowledge, IPV victims and without the decision-making power about health care tends to poor HIV knowledge. Supporting this result, Jasmin at el. ${ }^{30}$ describes that women with rights of decision-making power are affirmatively intended to have better HIV knowledge.

Social awarness against HIV needs to spread out whole over the country. Previous research has shown that the impact of peer-led HIV prevention programs to improve the knowledge of HIV/AIDS and motivates people to have better attitudes towards people living with $\mathrm{HIV}^{31,32}$ In some circumstances, teaching is the way of delivering a message for any communication. The improved period through the improved step of teaching delivers a chance to have more reproductive health evidence, more use of health maintenance facilities, and sustenance from noble clusters. ${ }^{14}$

\section{Limitations and strengths}

This study had several limitations. Firstly, as this is a sensitive matter, some respondents were hesitated to give data. Secondly, we used the data to perform analysis was from a secondary data source. So, a few important factors that were missing in this study. Nevertheless, we have tried to depict a compact illustration of the current situation, trend and determinants of HIV/AIDS knowledge in comparison of the urban and rural area. Moreover, only a very few studies were conducted in this area of study in Bangladesh.
Enhancing the available information hopefully, this research will contribute to developing suitable interventions for raising knowledge and awareness about HIV/AIDS among women especially in the rural area of Bangladesh.

\section{Conclusion}

The HIV/AIDS knowledge especially among women is a very crucial public health matter due to its significantly straight connection with HIV infection and the absence of wellbeing services and circuitously with death. In Bangladesh, women from rural residence have poor level of knowledge regarding HIV than the urban residents. The results revealed that the respondent's husband's education, economic and marital status, reading newspaper/radio, watching $\mathrm{TV}$, NGO health workers visit, women's decision-making power about health care are significantly associated with the knowledge about HIV/AIDS for both urban and rural women in Bangladesh. Alongside with these factors, age at marriage, condom use during intercourse, community clinic awareness, govt. health worker visits and victimization of IPV are also vital predictor of having HIV knowledge in rural Bangladesh.

To prevent HIV/AIDS, Bangladesh government should also consider encouraging the radio and TV channels to broadcast special programs that could increase the knowledge and consciousness among women about HIV/AIDS and improve the education intervention program by providing textbooks that contain more information on the sexual and reproductive system, especially for the primary and secondary schools. Also, peer-led HIV prevention programs should be implemented and frequent health worker visit should be ensured in this regard in rural area of Bangladesh.

\section{Ethics statement}

The Ministry of Health and Family Welfare, Dhaka, Bangladesh has given the ethical approval. The financial and technical assistance for the survey via USAID/Bangladesh was provided through the International Credit Finance (ICF). The worldwide Demographic and Health Surveys program is a part of the BDHS. ${ }^{20}$

\section{Competing Interests}

Authors declare that, they have no competing interests.

\section{Contributors' Statements}

MMAK is responsible for the study design and writing the manuscript. MSK, MGM and NT have done the formal analysis. MNIM and MRI contributed to drafting the manuscript and critically revising it for important intellectual content. All the authors read and approved the final manuscript.

\section{References}

1. Nubed CK, Akoachere JTK. Akoachere, Knowledge, attitudes and practices regarding HIV/AIDS among senior secondary school students in Fako Division, South West Region, Cameroon. BMC Public Health. 2016;16(1):847.

2. UNAIDS, Global HIV \& AIDS statistics- 2018 fact sheet. 2018;

3. WHO, World Health Organization | Data and statistics. WHO, 2018;

4. DGHS M. National HIV Serological Surveillance 2011. National AIDS/ STD Program. Directorate General Health Services (DGHS) and Ministry of Health and Family Welfare (MOHFW), Bangladesh. 2012; 
5. Stevens LM, Lynm C, Golub RM. Hiv infection: The basics. JAMA 2012;308(4):419.

6. Pendse R, Gupta S, Yu D, et al. HIV/AIDS in the South-East Asia region: progress and challenges. J Virus Erad. 2016;2(Suppl 4):1-6.

7. Sheikh MT, Uddin MN, Khan JR, et al. A comprehensive analysis of trends and determinants of HIV/AIDS knowledge among the Bangladeshi women based on Bangladesh Demographic and Health Surveys, 20072014. Archives of Public Health. 2017;75(1):59.

8. Hossain M, Mani KK, Sidik SM, et al. Knowledge and awareness abou STDs among women in Bangladesh. BMC Public Health. 2014;14(1):775.

9. Hossain MM, Sultana A, Mazumder H, et al. Sexually transmitted infections among Rohingya refugees in Bangladesh. The Lancet HIV. 2018;5(7):e342.

10. Babul B. Bangladesh is at high risk of HIV/AIDS epidemic. The Star Magazine. 2010;47.

11. Rahman MM, Kabir M, Shahidullah M, et al. Adolescent knowledge and awareness about AIDS/HIV and factors affecting them in Bangladesh. $J$ Ayub Med Coll Abbottabad. 2009;21(3):3-6.

12. Worldbank. HIV/AIDS in Bangladesh. 2012.

13. Hassan M. Encountering HIV/AIDS. Daily Star Web Ed. 2010;5:319325.

14. Hasan AH, Hassan R, Khan ZR, et al. Influence of socio-demographic factors on awareness of HIV/AIDS among Bangladeshi garment workers. Springerplus. 2013;2(1):174-178.

15. Mondal NI, Islam R, Rahman O, et al. Determinants of HIV/AIDS awareness among garments workers in Dhaka City, Bangladesh. World Journal of AIDS. 2012;2(04):312

16. Yaya S, Bishwajit G, Danhoundo G, et al. Trends and determinants of HIV/AIDS knowledge among women in Bangladesh. BMC Public Health. 2016;16(1):812.

17. Gubhaju BB. Adolescent reproductive health in Asia. Asia Pacific Population Journal. 2002;17(4):97-119.

18. Idele P, Gillespie A, Porth T, et al. Epidemiology of HIV and AIDS among adolescents: current status, inequities, and data gaps. JAIDS Journal of Acquired Immune Deficiency Syndromes. 2014;66(S2):S144-S153.

19. Ayodele O, Ayodele OM. Urban-Rural Differentials in HIV/AIDS Knowledge of Nigerian Senior Secondary School Students. International Journal of Health Sciences. 2016;4(3):35-41.

20. Niport M, ICF. Bangladesh Demographic and Health Survey 2004. National Institute of Population Research and Training (NIPORT), Dhaka, Bangladesh and ICF International. 2004.
21. Niport M, ICF. Bangladesh Demographic and Health Survey 2014. National Institute of Population Research and Training (NIPORT), Dhaka, Bangladesh and ICF International. 2014.

22. Appiah-Agyekum NN, Suapim RH. Knowledge and awareness of HIV AIDS among high school girls in Ghana. HIV/AIDS (Auckland, NZ). 2013;5:137.

23. Shokoohi M, Mohammad Karamouzian, Ali Mirzazadeh, et al HIV knowledge, attitudes, and practices of young people in Iran: findings of a national population-based survey in 2013. PloS one. 2016;11(9):e0161849.

24. Mondal MNI, Hoque N, Chowdhury MR, et al. Factors Associated with Misconceptions about HIV Transmission among Ever-Married Women in Bangladesh. Japanese Journal of Infectious Diseases. 2015;68(1):13-19.

25. Mondal MNI, Mahfuzar Rahman, Obaidur Rahman, et al. Level of awareness about HIV/AIDS among ever married women in Bangladesh. Food and Public Health. 2012;2(3):73-78.

26. Mondal NI, Takaku H, Ohkusa Y. Impact of age at marriage and migration on HIV and AIDS epidemics in Japan. International Journal for Equity in Health. 2009;8(1):23.

27. Khan MA. Knowledge on AIDS among female adolescents in Bangladesh evidence from the Bangladesh demographic and health survey data. Journal of health, population and nutrition. 2002;20(2):130-137.

28. Akelo V, Sonali Girde, Craig B Borkowf, et al. Attitudes toward family planning among HIV-positive pregnant women enrolled in a prevention of mother-to-child transmission study in Kisumu, Kenya. PloS one. 2013;8(8):e66593.

29. Jung M, Arya M, Viswanath K. Effect of Media Use on HIV/AIDSRelated Knowledge and Condom Use in Sub-Saharan Africa: A CrossSectional Study. PLoS ONE. 2013;8(7):e68359.

30. Jesmin SS, Rahman M. Social inequalities and the context of vulnerabilities: HIV/AIDS awareness and prevention knowledge among married women. Health care for women international. 2018;39(2):154-169.

31. Ibrahim N, Rampal L, Jamil Z, Zain AM. Effectiveness of peer-led education on knowledge, attitude and risk behavior practices related to HIV among students at a Malaysian public university - a randomized controlled trial. Preventive medicine. 2012;55(5):505-510.

32. Haroun D, El Saleh O, Wood L, et al. Assessing Knowledge of, and Attitudes to, HIV/AIDS among University Students in the United Arab Emirates. PLOS ONE. 2016;11(2):e0149920. 